\title{
Expression of amphiregulin predicts poor outcome in patients with pancreatic ductal adenocarcinoma
}

\author{
Li Wang, Huanwen Wu, Lili Wang, Junliang Lu, Huanli Duan, Xuguang Liu and Zhiyong Liang*
}

\begin{abstract}
Background: The validation of novel diagnostic, prognostic and predictive biomarkers in cancer is crucial for optimizing the choice and efficacy of personalized therapies. The aim of this study was to determine the epidermal growth factor receptor (EGFR), epidermal growth factor receptor variant III (EGFRvIII) and amphiregulin (AREG) protein expression levels and to evaluate the prognostic significance of EGFR, EGFRvIll and AREG in pancreatic ductal adenocarcinoma (PDAC).

Methods: The EGFR, EGFRvIll and AREG protein levels in PDAC $(n=92)$ were examined by using immunohistochemistry. The associations between EGFRvIll expression, AREG expression, AREG/EGFR co-expression and clinicopathological factors were assessed, the correlation between AREG and EGFR expression was analyzed and the survival analyses were performed.

Results: Among the lesions of PDAC, 12 (13\%) stained positive for EGFRvIll, 49 (53.3 \%) stained positive for AREG and 22(23.9\%) stained double positive for AREG/EGFR. The relationships between each protein expression level and the clinicopathologic factors were examined, only AREG/EGFR co-expression was significantly related to tumor differentiation $(P=0.032)$. The correlation between AREG and EGFR expression was statistically insignificant $(P=0.709)$. Univariate survival analysis proved that high tumor-node-metastasis (TNM) stage, poor tumor differentiation and AREG expression were significant poor prognostic factors for disease-free survival (DFS) and overall survival (OS). By multivariate survival analysis, tumor differentiation was an independent poor prognostic factor for DFS ( $H R=1.785, P<0.05)$, whereas high TNM stage $(H R=2.25, P<0.05)$, poor tumor differentiation ( $H R=2.125, P<0.01)$, positive resection margins $(H R=1.84, P<0.05)$, and AREG expression ( $H R=1.822, P<0.05)$ were all independent poor prognostic factors for OS.
\end{abstract}

Conclusions: In conclusion, our data indicate that AREG expression is an important prognostic biomarker in PDAC.

Keywords: EGFR, EGFRvIll, AREG, Pancreatic ductal adenocarcinoma

\section{Background}

Pancreatic ductal adenocarcinoma (PDAC) is the fourth leading cause of cancer-related deaths in the United States and the sixth leading cause of cancer-related deaths for males in China with a 5-year survival rate of less than $7 \%$ $[1,2]$. The majority of PDAC patients are diagnosed at an advanced stage and thus are not candidates for treatment with curative intent. Because PDAC patients usually show partial responses to traditional cytotoxic chemotherapy, specific molecule inhibition represents an attractive target

\footnotetext{
* Correspondence: liangzhiyong1220@yahoo.com

Molecular Pathology Research Center, Department of Pathology, Peking Union Medical College Hospital, Chinese Academy of Medical Sciences and Peking Union Medical College, Beijing 100730, People's Republic of China
}

for cancer therapy. The epidermal growth factor receptor (EGFR) has been increasingly recognized as a molecular target in cancer therapy. The combination of gemcitabine and erlotinib was the first combination therapy to demonstrate survival benefits in advanced or metastatic pancreatic cancer in a phase III, double-blind, placebo-controlled study [3]. As a result, gemcitabine-erlotinib combination therapy was approved by the Food and Drug Administration (FDA) for the first-line treatment of patients with locally advanced non-resectable or metastatic pancreatic cancer.

Dysregulated EGFR signaling (such as cell-surface overexpression, autocrine activation and EGFR gene mutation) contributes to the formation of several epithelial 
malignancies in humans [4]. There is increasing recognition that epidermal growth factor receptor variant III (EGFRvIII), the most common form of mutant EGFR, is an important target for cancer therapy. EGFRvIII comprises an in-frame deletion of 267 amino acids from the extracellular domain of EGFR. Although it is unable to bind ligand, EGFRvIII shows a low-level constitutive kinase activity and impaired endocytosis and degradation [5]. EGFRvIII is not detected in normal tissues, while it is overexpressed in several cancer types, particularly glioblastoma multiforme (GBM) [6]. However, whether EGFRvIII is expressed in pancreatic cancer remains unclear.

Amphiregulin (AREG) is a member of ligand family of EGFR. After bind to the extracellular ligand-binding domain of EGFR, AREG activates intracellular signaling cascades governing cell survival, proliferation, and motility [7]. Accordingly, several studies have focused on the disruption of AREG-mediated oncogenic pathways. AREG is upregulated in various neoplasms including colon, lung, liver, breast, prostate, and pancreatic cancer [7]. Functional studies show that AREG is involved in most of the hallmarks of cancer [8-11]. It has also been reported that AREG expression is a promising predictive marker for liver metastasis in primary colorectal cancer [9]. Tinhofer et al. demonstrated that patients with squamous cell carcinoma of the head and neck (SCCHN) who showed high AREG expression were less likely to benefit from combination treatment with cetuximab and docetaxel [10]. However, reports regarding AREG expression in pancreatic cancer by Park showed that decreased expression of AREG was a typical characteristic of the tumor biology [11].

Therefore, the aims of our study were to investigate the expression of EGFRvIII, AREG and AREG/EGFR coexpression in resected PDAC tissues and to explore the clinicopathological and prognostic significance of their expression in PDAC.

\section{Methods}

\section{Human tissues}

Patients who had preoperative chemotherapy (CT) or radiotherapy (RT), macroscopic incomplete resection (R2), or inadequate follow-up data and a survival time of less than 30 days from the time of surgery were excluded in our study. The study population comprised 92 patients who underwent the resection for PDAC at Peking Union Medical College Hospital during the period between January 2009 and December 2014. The study protocol was approved by the Institutional Ethics Committee at Peking Union Medical College Hospital. Written informed consent was obtained from all patients at the time of their treatment for use of material in future research.

\section{Clinicopathologic data}

The medical records of enrolled patients were reviewed from the pathologists' electronic medical records system at Peking Union Medical College Hospital. They included the following data: age, sex, date of surgery, tumor location, tumor size, pathologic stage (tumor-node-metastasis, TNM stage), tumor differentiation, patterns and the site of recurrence, patterns of resection margins. Disease free survival (DFS) was determined from the time from surgery until local or metastatic PDAC tumor recurrence. Overall survival (OS) was defined as the time of surgery to death. The follow-up period after the initial operation for primary lesions was between 1 to 5 years.

\section{Immunohistochemistry}

For the immunohistochemical study, 92 formalin-fixed, paraffin-embedded tumor specimens were collected. Conventional 4- $\mu \mathrm{m}$ sections from the tissue blocks were used. Immunohistochemistry was produced as previously described [12]. The slides were incubated with a monoclonal mouse anti-EGFR antibody (1:200 dilution; Santa Cruz), a monoclonal mouse anti-EGFRvIII antibody (1:200 dilution; Biorbyt), or a polyclonal goat anti-AREG antibody (1:50 dilution; R\&D).

\section{Evaluation of immunostaining}

For the membranous and/or cytoplasmic expression levels of EGFR and EGFRvIII, immunoreactivity was defined in the same manner as previously described [13]. The cytoplasmic expression levels of AREG was socred by applying a semi-quantitative immunoreactive score (IRS) system [14]. Briefly, immunostatining intensity was scored as: $0=$ no staining, $1=$ weak staining, $2=$ moderate staining and 3 $=$ strong staining. The extent of stained cells was stratified into three groups based on the percentage of positive cells: $0=0 \%, 1=1-33 \%, 2=33-66 \%$; and $3=>66 \%$. IRS scores were obtained by multiplying the staining intensity by the number of group which ranging from 0 to 9 . The final IRS scores $>3$ were considered AREG positive. The slides were independently evaluated by two of the authors (LW and HWW) to assess the protein expression levels.

\section{Statistical analysis}

The categorical variables were compared using the $\chi^{2}$ test. The correlation between AREG and EGFR expression was examined by Pearson's test. Kaplan-Meier survival curves were used to estimate the disease-free survival (DFS) and overall survival (OS) of the patients which was determined using the log-rank test. Multivariate analysis was performed by Cox proportional hazard regression mode. Two-sided $P$ values $<0.05$ were considered significant. All statistical procedures were performed with SPSS software for Windows, version 17.0 (SPSS Inc., Chicago, IL, USA). 
Table 1 Summary of baseline patient characteristics $(n=92)$

\begin{tabular}{lll}
\hline Characteristic & Variable & Value \\
\hline Age range (years) & & $34-80$ \\
& Median & 61 \\
& Mean & 60 \\
Sex & Male vs. Female & $50: 42$ (54.3 vs. $45.7 \%)$ \\
Tumor location & Head vs. Body/tail & $52: 40$ (56.5 vs. $43.5 \%)$ \\
Tumor size & T1-2 vs. T3-4 & $23: 69$ (25 vs. $75 \%)$ \\
TNM stage & I-II vs. III-IV & $80: 12$ (87 vs. $13 \%)$ \\
Tumor differentiation & Well/moderate vs. Poor & $63: 29(68.5$ vs. $31.5 \%)$ \\
Lymph node metastasis & Yes vs. No & $51: 41$ (55.4 vs. $44.6 \%)$ \\
Resection margins & Positive vs. Negative & $18: 74$ (20 vs. $80 \%)$ \\
\hline
\end{tabular}

\section{Results}

Patient characteristics and Immunohistochemical analysis Among the 92 PDAC patients, there were 50 men and 42 women with a median age of 61 years (range: $34-80$ years). The patient characteristics were described in Table 1 .

In total, EGFRvIII expression of PDACs was 12 (13\%), AREG expression of PDACs was 49 (53.3 \%) and AREG/
EGFR co-expression of PDACs was22 (23.9 \%). EGFR and EGFRvIII was predominantly localized at the cellular membrane, AREG were mainly detected in the cytoplasm. Representative PDAC tissues with EGFR, EGFRvIII and AREG expression profiles are shown in Fig. 1. The acinar and ductal cells of the peritumoral areas showed negative or weak staining for EGFR and AREG, whereas these proteins were weakly or moderately expressed in normal pancreatic islet cells. Weak or moderate staining for AREG was also observed in a majority of the fibroblasts.

A significant association between AREG/EGFR coexpression and tumor differentiation was observed in our study $(P=0.032)$. However, there was no significant association between EGFRvIII expression or AREG expression alone and clinicopathological characteristics in PDAC, (Table 2).

\section{Correlation between AREG and EGFR expression}

Among 92 PDAC patients, 22 were positive for AREG and EGFR expression, 21 were single-positive for EGFR, 27 were single-positive for AREG, and 22 were negative for AREG and EGFR. The correlation between EGFR and
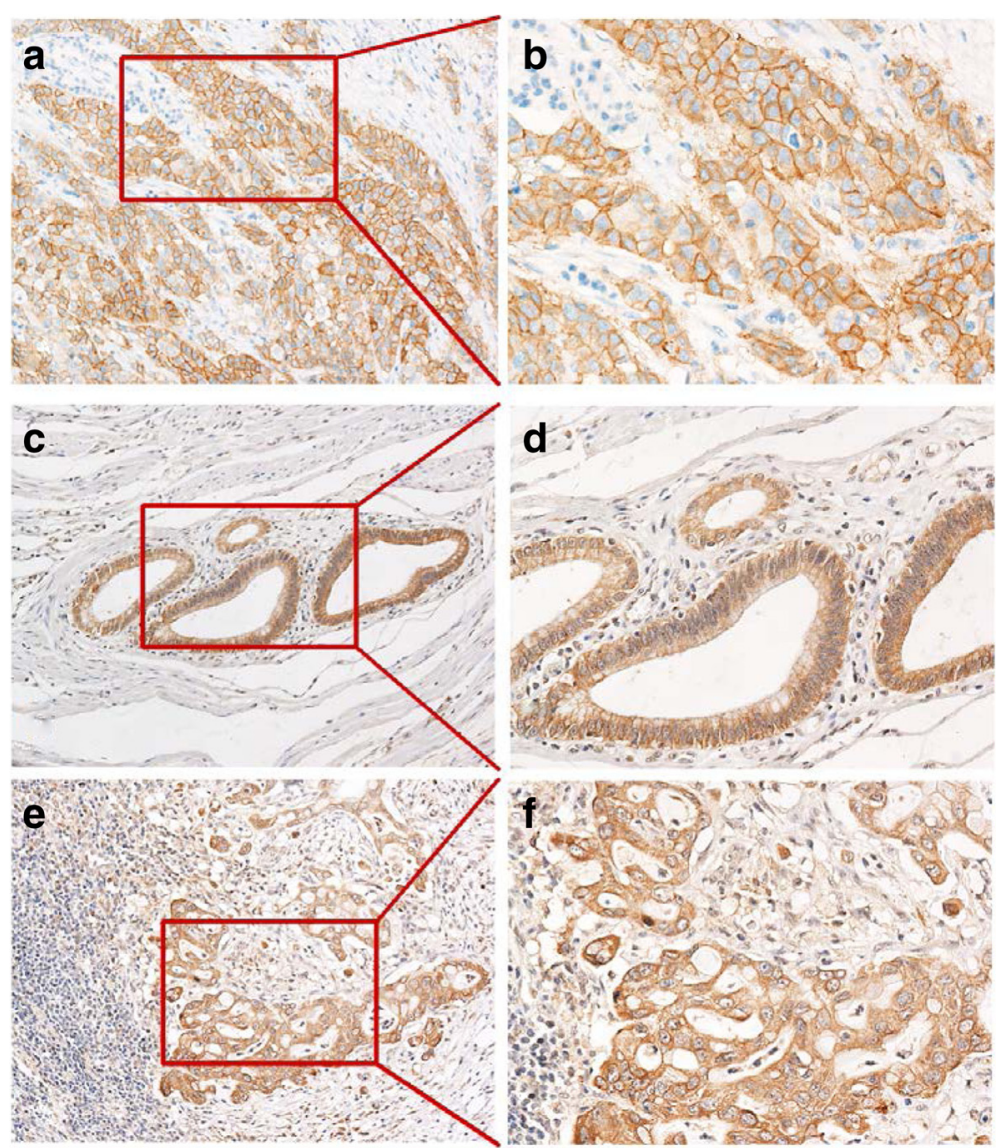

Fig. 1 Detection of EGFR, EGFRvIII, AREG expression in PDAC. a representative tumor samples with EGFR expression, $\times 100$. $\mathbf{b}$ EGFR expression, ×200. c EGFRvIII expression, ×100. d EGFRvIII expression, ×200. e AREG expression, ×100. f AREG expression, ×200 
Table 2 Correlation between EGFRvIll expression, AREG expression, AREG/EGFR co-expression and clinicopathologic factors in PDAC

\begin{tabular}{|c|c|c|c|c|c|c|c|c|c|}
\hline \multirow[t]{2}{*}{ Parameter } & \multicolumn{2}{|l|}{ EGFRvIII } & \multirow[t]{2}{*}{$P$} & \multicolumn{2}{|l|}{ AREG } & \multirow[t]{2}{*}{ P } & \multicolumn{2}{|c|}{ AREG/EGFR } & \multirow[t]{2}{*}{$P$} \\
\hline & Negative & $\overline{\text { Positive }}$ & & Negative & $\overline{\text { Positive }}$ & & Negative $^{a}$ & Positive $^{b}$ & \\
\hline Overall & 80 & 12 & & 43 & 49 & & 70 & 22 & \\
\hline Age(years) & & & 0.056 & & & 0.241 & & & 0.65 \\
\hline$<60$ & 30 & 8 & & 15 & 23 & & 28 & 10 & \\
\hline$\geq 60$ & 50 & 4 & & 28 & 26 & & 42 & 12 & \\
\hline Gender & & & 0.124 & & & 0.566 & & & 0.057 \\
\hline Male & 39 & 3 & & 22 & 28 & & 34 & 16 & \\
\hline Female & 41 & 9 & & 21 & 21 & & 35 & 6 & \\
\hline Tumor sites & & & 0.625 & & & 0.256 & & & 0.09 \\
\hline Head & 46 & 6 & & 27 & 25 & & 43 & 9 & \\
\hline Body/tail & 34 & 6 & & 16 & 24 & & 27 & 13 & \\
\hline Tumor size & & & 0.475 & & & 0.117 & & & 0.158 \\
\hline T1-2 & 21 & 2 & & 14 & 9 & & 20 & 3 & \\
\hline T3-4 & 59 & 10 & & 29 & 40 & & 50 & 19 & \\
\hline TNM stage & & & 0.187 & & & 0.106 & & & 0.925 \\
\hline$|-| \mid$ & 71 & 9 & & 40 & 40 & & 61 & 19 & \\
\hline III-IV & 9 & 3 & & 3 & 9 & & 12 & 3 & \\
\hline Tumor differentiation & & & 0.885 & & & 0.803 & & & 0.032 \\
\hline Well/morderate & 55 & 8 & & 30 & 33 & & 52 & 11 & \\
\hline Poor & 25 & 4 & & 13 & 16 & & 18 & 11 & \\
\hline Resection margins & & & 0.067 & & & 0.457 & & & 0.851 \\
\hline Negative & 62 & 12 & & 36 & 38 & & 56 & 18 & \\
\hline Positive & 18 & 0 & & 7 & 11 & & 14 & 4 & \\
\hline Lymph node metastasis & & & 0.828 & & & 0.725 & & & 0.923 \\
\hline No & 36 & 5 & & 20 & 21 & & 31 & 10 & \\
\hline Yes & 44 & 7 & & 23 & 28 & & 39 & 12 & \\
\hline
\end{tabular}

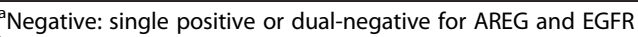

${ }^{b}$ Positive: AREG/EGFR coexpression(double positive for AREG and EGFR)

AREG expression was statistically insignificant $(\Phi=0.039$, $P=0.709)$.

\section{Prognostic Factors Affecting DFS and OS}

The median follow-up for DFS and OS was 9.50 months (range, 1-36 months) and 17.50 months (range, 2-48 months), respectively. The mean DFS and OS was 11.47 months and 17.71 months, respectively.

In the univariate survival analysis, high TNM stage $(P=0.003$ and $P=0.001)$, poor tumor differentiation $(P=0.009$ and $P=0.002)$ and AREG expression $(P=0.021$ and $P=0.003)$ were significant adverse prognostic factors for DFS and OS, respectively (Table 3, Fig. 2). PDAC patients with positive expression for AREG showed significantly shorter DFS and OS (AREG-positive, 8 months vs. AREG-negative, 12 months; AREG-positive, 16 months vs. AREG-negative, 23 months). To analyze the prognostic significance of the AREG/EGFR interaction, the study population was divided into three groups: an AREG/EGFR co-expression group, a single-positive group and a dualnegative group. Using the log-rank test, however, none of these groups showed significant differences in DFS or OS (data not shown).

The hazard ratio (HR) was estimated by Cox regression, AREG expression was found to increase the HR for recurrence $(\mathrm{HR}=1.688,1.059-2.691)$ and death $(\mathrm{HR}=$ 2.043, 1.238-3.374) (Table 3). Multivariate analysis was performed to find independent factors that could affect DFS and OS. Factors at the 0.10 level in the univariate analysis (TNM stage, tumor differentiation, tumor size, Resection margins, lymph node metastasis, AREG expression and AREG/EGFR co-expression) were entered into a multivariate survival analysis. Poor tumor differentiation was an independent unfavorable prognostic factor for DFS $(\mathrm{HR}=1.785, P=0.021)$ and $\mathrm{OS}(\mathrm{HR}=$ 2.125, $P=0.004)$. Moreover, AREG expression $(\mathrm{HR}=$ 1.822, $P=0.03)$, high TNM stage $(\mathrm{HR}=225, P=0.03)$, and positive resection margins $(\mathrm{HR}=1.84, P=0.045)$ 
Table 3 Univariate analysis for disease-free survival and overall survival

\begin{tabular}{|c|c|c|c|c|c|c|}
\hline \multirow[t]{2}{*}{ Parameter } & \multicolumn{3}{|l|}{ DFS } & \multicolumn{3}{|l|}{ OS } \\
\hline & $\mathrm{HR}$ & $95 \% \mathrm{Cl}$ & $P$ & $\mathrm{HR}$ & $95 \% \mathrm{Cl}$ & $P$ \\
\hline Age(years) & & & 0.944 & & & 0.84 \\
\hline$\geq 60$ (vs. <60) & 0.985 & $0.632-1.537$ & & 0.954 & $0.594-1.534$ & \\
\hline Gender & & & 0.456 & & & 0.416 \\
\hline Female (vs. Male) & 1.176 & $0.754-1.835$ & & 1.21 & $0.754-1.943$ & \\
\hline Tumor sites & & & 0.69 & & & 0.629 \\
\hline Body/tail (vs. Head) & 0.918 & $0.589-1.431$ & & 0.893 & $0.555-1.439$ & \\
\hline Tumor size & & & 0.181 & & & 0.052 \\
\hline T3-4 (vs. T1-2) & 1.379 & $0.836-2.274$ & & 1.658 & $0.967-2.843$ & \\
\hline TNM stage & & & 0.003 & & & 0.001 \\
\hline III-IV (vs. I-II) & 2.399 & $1.284-4.482$ & & 2.849 & $1.428-5.683$ & \\
\hline Tumor differentiation & & & 0.009 & & & 0.002 \\
\hline Poor (vs. Well/moderate) & 1.784 & $1.118-2.846$ & & 2.059 & $1.269-3.34$ & \\
\hline Resection margins & & & 0.282 & & & 0.089 \\
\hline Positive (vs. Negative) & 1.338 & $0.77-2.326$ & & 1.601 & $0.912-2.81$ & \\
\hline Lymph node metastasis & & & 0.314 & & & 0.06 \\
\hline Yes (vs. No) & 1.238 & $0.797-1.923$ & & 1.535 & $0.961-2.453$ & \\
\hline AREG expression & & & 0.021 & & & 0.003 \\
\hline Positive (vs. Negative) & 1.688 & $1.059-2.691$ & & 2.043 & $1.238-3.374$ & \\
\hline EGFRvIII expression & & & 0.466 & & & 0.466 \\
\hline Positive (vs. Negative) & 1.256 & $0.663-2.381$ & & 1.292 & $0.637-2.623$ & \\
\hline AREG/EGFR coexpression & & & 0.08 & & & 0.166 \\
\hline Negative (vs. Positive) & 0.853 & $0.646-1.128$ & & 0.831 & $0.619-1.115$ & \\
\hline
\end{tabular}

were all independent prognostic indicators for poor OS (Table 4).

\section{Discussion}

Unfortunately, PDAC is associated with a largely unfavorable outcome and aggressive tumor biology. Tumor size, lymph node involvement and the status of the resection margin are traditional prognostic factors, although they are not adequate to distinguish between patients with a high and low risk of disease recurrence and metastasis. Our study is the first to simultaneously investigate the clinicopathological and prognostic significance of EGFRvIII (the most common mutated variant of EGFR) expression, AREG (EGFR ligand) expression and AREG/EGFR co-expression in PDAC. In our study, we showed that AREG/EGFR co-expression were associated with poor tumor differentiation. Also, AREG expression in PDAC was an independent prognostic indicator of poor OS according to our multivariate survival analysis.

According to previous immunohistochemical studies, EGFR is expressed in 23.9-68.4\% of PDAC samples [12, 15]. Handra et al. [16] found that tumor expression of EGFR was associated with clinical response but not outcome in PDAC. Funatomi et al. demonstrated the existence of an autoregulated AREG/EGFR feedback loop in pancreatic cancer [17]. After binding to EGFR, AREG stimulation of the intrinsic tyrosine kinase activity of EGFR induces a complex cascade of phosphorylation and activation events that determine cell proliferation, differentiation, and tumor development [18]. We found that the expression of AREG/ EGFR co-expression were associated with poor tumor differentiation, which is consistent with previous studies.

In recent years, investigators have increasingly recognized the critical role of EGFRvIII in tumor carcinogenesis [19-21]. To the best of our knowledge, no published study has correlated the expression of EGFRvIII with prognosis in patients with pancreatic cancer. Evaluation of the expression of EGFRvIII in PDAC could provide additional knowledge concerning the complex mechanism of EGFR signaling in PDAC. EGFRvIII expression occurs at an overall frequency of 25-64\% when assessed by multiple techniques in GBM [20, 21]. Using immunohistochemistry, our study showed that $13 \%$ (12 of 92) of PDAC patients were positive for EGFRvIII. Tinhofer et al. [10] found that expression of EGFRvIII was detected in $17 \%$ of SCCHN patients, expression of EGFRvIII was significantly associated with shortened PFS but not with OS. In GBM, EGFRvIII expression was associated with poor prognosis 

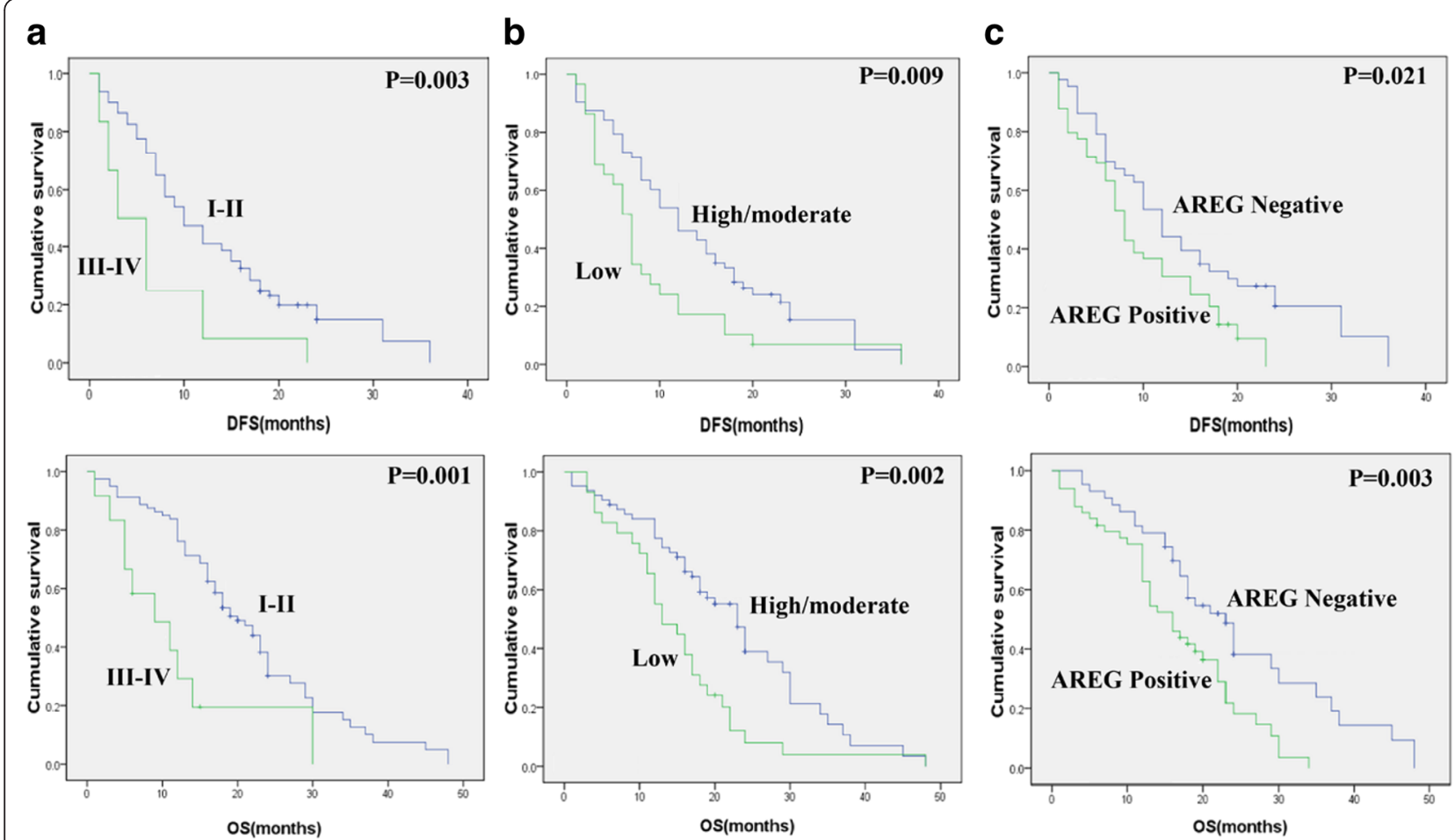

Fig. 2 Predictors of DFS and OS in PDAC. Kaplan-Meier curves for DFS (top) and OS (bottom) of PDAC patients with TNM stage (a), Tumor differentiation (b) and AREG expression (c). P values for comparison of groups using the log-rank test are given

$[20,21]$. However, our study suggests that EGFRvIII expression has little prognostic significance on survival in PDAC patients. Thus, the prognostic significance of EGFRvIII expression in PDAC remains unclear and requires further clarification.

AREG has been recognized as an oncogenic factor for more than 20 years. The role of AREG in cancer development and progression is supported by clinical data showing that AREG can serve as a prognostic and/or a predictive biomarker [22-26]. Masago et al. [24] found that high serum levels of AREG and TGF- $\alpha$ were predictors of poor prognosis in patients with advanced nonsquamous, non-small cell lung cancer (NSCLC). Similarly, AREG expression was shown to be independently associated with a reduced OS in a multivariate analysis of 195 patients with stages I-III NSCLC [25]. Moreover, the concomitant expression of AREG and EGFR was associated with enhanced tumor aggressiveness and shorter survival periods following tumor resection in PDAC [26]. The results from in vitro and in vivo studies have demonstrated that aberrantly activated AREGEGFR signaling is required for CRTC1-MAML2-positive MEC (mucoepidermoid carcinoma) cell growth and survival. In particular, CRTC1-MAML2-positive MEC cells are highly sensitive to EGFR signaling inhibition, which suggests that EGFR-targeted therapies may benefit patients with MEC [27]. Consistent with these results, our study demonstrated that high AREG expression was an independent prognosticator of poor OS in PDAC. We also found that AREG/EGFR co-expression was associated with poor tumor differentiation, which is similar to that demonstrated in previous studies [22-26]. Recently, one study with conflicting results reported that the negative expression of AREG and positive expression of MMP-2 were hallmarks of tumor biology in PDAC patients [11]. Park et al. enrolled 88 PDAC patients and stained EGFR, AREG, VEGF, p-c-met, MMP2, MMP7, MMP9, CXCR3, and CXCR4 antibodies on tissue microarray (TMA) [11]. On the contrary, we stained our markers on whole tissue sections, which provided a wider scope and improved perception of tumor heterogeneity, eliminating potential bias. Compared with their uniform interpretation criteria, we adopted different criteria for positivity membranous staining and cytoplasmic staining. However, both studies were subjected to several limitations including limited sample size and lack the in-depth investigation into the prooncogenic mechanism of AREG in PDAC.

Numerous researchers have reported that AREG was correlated with invasion and distant metastases in multiple malignancies [28-30]. Increasing evidences revealed that the essential role of EMT in the local invasion and metastasis of pancreatic cancer. To study the role of AREG in the EMT of PDAC may provide insights into the tumor biology of PDAC cell migration and invasion for inspiration. 
Table 4 Multivariate analysis for disease-free survival and overall survival

\begin{tabular}{|c|c|c|c|}
\hline Parameter & $\mathrm{HR}$ & $95 \% \mathrm{Cl}$ & $P$ \\
\hline \multicolumn{4}{|c|}{ Disease-free survival: Cox regression model } \\
\hline TNM stage & & & 0.06 \\
\hline III-IV (vs. I-II) & 2.528 & $1.299-4.921$ & \\
\hline Tumor differentiation & & & 0.021 \\
\hline Poor (vs. Well/moderate) & 1.785 & $1.089-2.925$ & \\
\hline AREG expression & & & 0.168 \\
\hline Positive (vs. Negative) & 1.418 & $0.863-2.331$ & \\
\hline AREG/EGFR coexpression & & & 0.587 \\
\hline Negative (vs. Positive) & 0.922 & $0.687-1.237$ & \\
\hline \multicolumn{4}{|c|}{ Overall survival: Cox regression model } \\
\hline Tumor size & & & 0.485 \\
\hline T3-4 (vs. T1-2) & 1.24 & $0.677-2.271$ & \\
\hline TNM stage & & & 0.03 \\
\hline III-IV (vs. I-II) & 2.25 & $1.081-4.684$ & \\
\hline Tumor differentiation & & & 0.004 \\
\hline Poor (vs. Well/moderate) & 2.125 & $1.277-3.537$ & \\
\hline Resection margins & & & 0.045 \\
\hline Positive (vs. Negative) & 1.84 & $1.277-3.537$ & \\
\hline Lymph node metastasis & & & 0.417 \\
\hline Yes (vs. No) & 1.233 & $0.743-2.048$ & \\
\hline AREG expression & & & 0.03 \\
\hline Positive (vs. Negative) & 1.822 & $1.058-3.137$ & \\
\hline
\end{tabular}

\section{Conclusions}

Our results showed that although no significant association was observed between AREG expression alone and the clinicopathological characteristics in PDAC, there was a significant association between AREG/EGFR co-expression and poor tumor differentiation. Moreover, AREG expression, poor tumor differentiation, high TNM stage, and positive resection margins were all independent prognostic indicators for poor OS. Our data indicate that AREG expression identifies a subset of PDAC patients with more aggressive tumor characteristics and a significantly worse prognosis, indicating that AREG may serve as an attractive therapeutic strategy for PDAC.

\section{Additional file}

Additional file 1: Figure S1. AREG RNA and protein levels in pancreatic cancer cells and pancreatic stellate cells. A, AREG mRNA levels in pancreatic cancer cells and pancreatic stellate cells. B, AREG protein levels in pancreatic cancer cells and pancreatic stellate cells, ${ }^{*}$ indicates a $P<0.05$. (DOC $718 \mathrm{~kb}$ )

\section{Abbreviations}

EGFR, epidermal growth factor receptor; EGFRvIll, epidermal growth factor receptor variant III; AREG, amphiregulin; PDAC, pancreatic ductal adenocarcinoma; GBM, glioblastoma multiforme; SCCHN, squamous cell carcinoma of the head and neck; NSCLC, non-small cell lung cancer; $\subset$, chemotherapy; RT, radiotherapy; DFS, disease-free survival; OS, overall survival

Acknowledgements

Not applicable.

\section{Funding}

This work was supported by the Special Foundation for Scientific Research in the Public Interest by the National Health and Family Planning Commission of China (201402001), National Natural Science Foundation of China

(31471366), PUMC Youth Fund (A107000) and Specialized Research Fund for the Doctoral Program of Higher Education of China (81150027).

\section{Availability of data and materials}

The datasets supporting the conclusions of this article are included within the article and its Additional file 1.

\section{Authors' contributions}

$L W, H D$, JL and $X L$ performed the experiments, LW and HW performed the statistical analysis, LW and HW wrote the paper, ZL conceived of the study, and participated in its design and coordination and helped to draft the manuscript. All authors read and approved the final manuscript.

\section{Competing interests}

The authors have no competing interests to disclose.

\section{Consent for publication}

Not applicable.

\section{Ethics approval and consent to participate}

The study protocol was approved by the Institutional Ethics Committee at Peking Union Medical College Hospital (No. S-K118). Written informed consent was obtained from all patients at the time of their treatment for use of material in future research.

Received: 18 December 2015 Accepted: 2 July 2016

Published online: 08 July 2016

\section{References}

1. Siegel RL, Miller KD, Jemal A. Cancer statistics, 2015. CA Cancer J Clin. 2015; 65:5-29. doi:10.3322/caac.21254.

2. Chen W, Zheng R, Baade PD, Zhang S, Zeng H, Bray F, et al. Cancer statistics in China, 2015. CA Cancer J Clin. 2016;66(2):115-32. doi:10.3322/caac.21338. Epub 2016 Jan 25.

3. Moore MJ, Goldstein D, Hamm J, Figer A, Hecht JR, Gallinger S, et al. Erlotinib plus gemcitabine compared with gemcitabine alone in patients with advanced pancreatic cancer: a phase III trial of the National Cancer Institute of Canada Clinical Trials Group. J Clin Oncol. 2007;25(15):1960-6.

4. Arteaga $\mathrm{CL}$. Epidermal growth factor receptor dependence in human tumors: more than just expression? Oncologist. 2002;7 Suppl 4:31-9. doi:10.1634/theoncologist.7-suppl_4-31.

5. Gan HK, Kaye AH, Luwor RB. The EGFRvIll variant in glioblastoma multiforme. J Clin Neurosci. 2009;16:748-54. doi:10.1016/j.jocn.2008.12.005.

6. Gan HK, Burgess AW, Clayton AHA, Scott AM. Targeting of a conformationally exposed, tumor-specific Epitope of EGFR as a strategy for cancer therapy. Cancer Res. 2012;72(12):2924-30. doi:10.1158/0008-5472.CAN-11-3898.

7. Berasain C, Avila MA. Amphiregulin. Semin Cell Dev Biol. 2014;28:31-41. doi:10.1016/j.semcdb.2014.01.005.

8. Busser B, Sancey L, Brambilla E, Coll JL, Hurbin A. The multiple roles of amphiregulin in human cancer. Biochim Biophys Acta. 2011;1816(2):119-31. doi:10.1016/j.bbcan.2011.05.003.

9. Yamada M, Ichikawa Y, Yamagishi S, Momiyama N, Ota M, Fujii S, et al. Amphiregulin is a promising prognostic marker for liver metastases of colorectal cancer. Clin Cancer Res. 2008;14:2351-6. doi:10.1158/1078-0432.CCR-07-4499.

10. Tinhofer I, Klinghammer K, Weichert W, Knodler M, Stenzinger A, Gauler $T$, et al. Expression of Amphiregulin and EGFRvIll affect outcome of patients with squamous cell carcinoma of the head and neck receiving Cetuximab-Docetaxel treatment. Clin Cancer Res. 2011;17:5197-204. doi: 10.1158/1078-0432.CCR-10-3338.

11. Park JK, Kim MA, Ryu JK, Yoon YB, Kim S, Han H, et al. Postoperative prognostic predictors of pancreatic ductal adenocarcinoma: clinical analysis 
and immunoprofile on tissue microarrays. Ann Surg Oncol. 2012;19:2664-72. doi:10.1245/s10434-012-2277-7.

12. Wu H, Zhu L, Zhang H, Shi X, Zhang L, Wang W, et al. Co-expression of EGFR and CXCR4 predicts poor prognosis in resected pancreatic ductal adenocarcinoma. PLoS One. 2015;10:e0116803.

13. Shinojima N, Tada K, Shiraishi S, Kamiryo T, Kochi M, Nakamura H, et al. Prognostic value of epidermal growth factor receptor in patients with glioblastoma multiforme. Cancer Res. 2003;63(20):6962-70.

14. Nagathihalli NS, Beesetty Y, Lee W, Washington MK, Chen X, Lockhart AC, et al. Novel mechanistic insights into Ectodomain shedding of EGFR ligands Amphiregulin and TGF-a: impact on gastrointestinal cancers driven by secondary bile acids. Cancer Res. 2014;74:2062-72. doi:10.1158/0008-5472.CAN-13-2329.

15. Luo G, Long J, Qiu L, Liu C, Xu J, Yu X. Role of epidermal growth factor receptor expression on patient survival in pancreatic cancer: A Metaanalysis. Pancreatology. 2011;11:595-600. doi:10.1159/000334465.

16. Handra-Luca A, Hammel P, Sauvanet A, Lesty C, Ruszniewski P, Couvelard A. EGFR expression in pancreatic adenocarcinoma. Relationship to tumour morphology and cell adhesion proteins. J Clin Pathol. 2014;67:295-300. doi:10.1136/jclinpath-2013-201662.

17. Funatomi H, Itakura J, Ishiwata T, Pastan I, Thompson SA, Johnson GR, et al. Amphiregulin antisense oligonucleotide inhibits the growth of T3M4 human pancreatic cancer cells and sensitizes the cells to EGF receptor-targeted therapy. Int J Cancer. 1997;72:512-7. doi:10.1002/(SICI) 1097-0215(19970729)72:3<512:AIDIJC21>3.0.CO;2-C

18. Avraham $R$, Yarden Y. Feedback regulation of EGFR signalling: decision making by early and delayed loops. Nat Rev Mol Cell Biol. 2011;12:104-17. doi:10.1038/ nrm3048.

19. Gan HK, Cvrljevic AN, Johns TG. The epidermal growth factor receptor variant III (EGFRvIII): where wild things are altered. FEBS J. 2013;280:5350-70. doi:10.1111/febs.12393.

20. Saikali S, Avril T, Collet B, Hamlat A, Bansard J, Drenou B, et al. Expression of nine tumour antigens in a series of human glioblastoma multiforme: interest of EGFRvIII, IL-13Ralpha2, gp100 and TRP-2 for immunotherapy. J Neurooncol. 2006;81:139-48. doi:10.1007/s11060-006-9220-3.

21. Heimberger AB, Hlatky R, Suki D. Prognostic effect of epidermal growth factor receptor and EGFRvIll in glioblastoma multiforme patients. Clin Cancer Res. 2005;11:1462-6. doi:10.1158/1078-0432.CCR-04-1737.

22. Addison CL, Ding K, Zhao H, Le Maître A, Goss GD, Seymour L, et al. Plasma transforming growth factor-a and amphiregulin protein levels in NSCLC Clinical Trials Group. J Clin Oncol. 2010;21:5247-56.

23. Ishikawa N, Daigo Y, Takano A. Increases of amphiregulin and transforming growth factor-alpha in serum as predictors of poor response to gefitinib among patients with advanced non-small cell lung cancers. Cancer Res. 2005;65:9176-84. doi:10.1158/0008-5472.CAN-05-1556.

24. Masago K, Fujita S, Hatachi Y, Fukuhara A, Sakuma K, Ichikawa M, et al. Clinical significance of pretreatment serum amphiregulin and transforming growth factor-a, and an epidermal growth factor receptor somatic mutation in patients with advanced non-squamous, non-small cell lung cancer. Cancer Sci. 2008;99:2295-301. doi:10.1111/j.1349-7006.2008.00931.x.

25. Fontanini $G$, De Laurentiis $M$, Vignati $S$, Chinè $S$, Lucchi $M$, Silvestri $V$, et al. Evaluation of epidermal growth factor-related growth factors and receptors and of neoangiogenesis in completely resected stage I-IIIA non-small-cell lung cancer: amphiregulin and microvessel count are independent prognostic indicators of survival. Clin Cancer Res. 1998;4(1):241-9.

26. Kleeff J, Friess H, Berberat PO, Martignoni ME, Z'graggen K, Büchler MW. Pancreatic cancer-new aspects of molecular biology research. Swiss Surg. 2000;6:231-4. doi:10.1024/1023-9332.6.5.231

27. Chen Z, Chen J, Gu Y, Hu C, Li J, Lin S, et al. Aberrantly activated AREGEGFR signaling is required for the growth and survival of CRTC1-MAML2 fusion-positive mucoepidermoid carcinoma cells. Oncogene. 2014;33:386977. doi:10.1038/onc.2013.348.

28. So WK, Fan Q, Lau MT, Qiu X, Cheng JC, Leung PC. Amphiregulin induces human ovarian cancer cell invasion by down-regulating E-cadherin expression. FEBS Lett. 2014;588(21):3998-4007. doi:10.1016/jffebslet.2014.09.017. Epub 2014 Sep 23.

29. Saeki M, Egusa H, Kamano Y, Kakihara Y, Houry WA, Yatani H, et al. Exosome-Bound WD repeat protein monad inhibits breast cancer cell invasion by Degrading Amphiregulin mRNA. PLoS One. 2013;8(7):e67326. doi:10.1371/journal.pone.0067326.

30. Higginbotham JN, Demory Beckler M, Gephart JD, Franklin JL, Bogatcheva G, Kremers GJ, et al. Amphiregulin exosomes increase cancer cell invasion. Curr Biol. 2011;21(9):779-86. doi:10.1016/j.cub.2011.03.043. Epub 2011 Apr 21.

\section{Submit your next manuscript to BioMed Central and we will help you at every step:}

- We accept pre-submission inquiries

- Our selector tool helps you to find the most relevant journal

- We provide round the clock customer support

- Convenient online submission

- Thorough peer review

- Inclusion in PubMed and all major indexing services

- Maximum visibility for your research

Submit your manuscript at www.biomedcentral.com/submit
(O) BioMed Central 